\title{
Filigrane
}

Écoutes psychanalytiques

\section{Regard, haine et emprise dans une relation mère-fils. Un commentaire du film We Need to Talk about Kevin de Lynne Ramsay (2011)}

\section{Réal Laperrière}

Volume 24, numéro 2, automne 2015

URI : https://id.erudit.org/iderudit/1036539ar

DOI : https://doi.org/10.7202/1036539ar

Aller au sommaire du numéro

Éditeur(s)

Revue Santé mentale au Québec

ISSN

1192-1412 (imprimé)

1911-4656 (numérique)

Découvrir la revue

Citer ce document

Laperrière, R. (2015). Regard, haine et emprise dans une relation mère-fils. Un commentaire du film We Need to Talk about Kevin de Lynne Ramsay (2011)

Filigrane, 24(2), 203-213. https://doi.org/10.7202/1036539ar d'utilisation que vous pouvez consulter en ligne. 


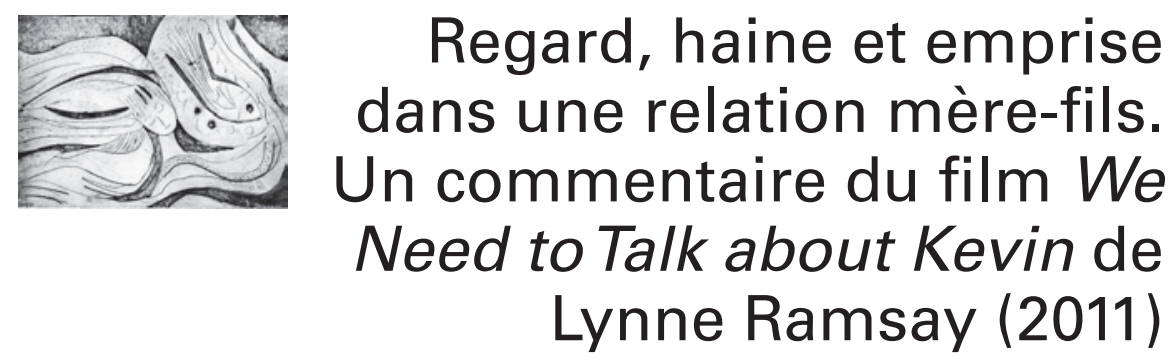

Réal Laperrière

L'enfant doit être amené, par une prodigieuse dépense d'amour, de tendresse et de soins, à pardonner aux parents de l'avoir mis au monde sans lui demander son intention, sinon les pulsions de destruction se meuvent aussitôt.

S. Ferenczi, $1929^{1}$

«D uisque tu t'intéresses depuis longtemps aux enfants difficiles». Tel était l'argument de l'organisateur du Ciné-Psy ${ }^{2}$ pour me convaincre de commenter We Need to Talk about Kevin. Ma réaction spontanée a été négative, et pour une unique raison: si j'acceptais, il me faudrait revoir ce film. C'est dire à quel point j'avais été troublé par mon premier visionnage, quelques années auparavant, voire même effrayé, et que je ne pouvais ressentir qu'une grande appréhension à l'idée de me retrouver à nouveau enfermé dans cette relation mère-fils étouffante et passionnée, cette relation passionnément haineuse, à laquelle même le plus horrible des évènements ne pourrait mettre un terme.

Lorsque l'actualité nous confronte à des cas aussi sordides et monstrueux que celui de Kevin Khatchadourian (et les dernières années au Québec nous en ont fourni quelques-uns), on est souvent porté à se poser en "expert psycho-légal»: on a tous alors notre opinion clinique sur le «cas», notre diagnostic, psychiatrique, psychopathologique, psychosocial, sociologique ou autre; diagnostic qu'on peut confronter à tous ceux que posent dans les médias la multitude de spécialistes sollicités, ainsi que les experts «spontanés» qui s'y expriment avec une assurance déconcertante. Après tout, devant tant d'horreur à recevoir en soi et à traiter psychiquement, on ne peut éviter de chercher à comprendre. Pour objectiver, mettre hors de soi, se rassurer 
un tant soit peu. Et tenter de nier toute identification aux protagonistes du drame. Or, ce n'est pas la voie que j'ai choisie pour mon commentaire. Je ne chercherai donc pas tant à faire un «diagnostic» de Kevin, de ses parents ou de son système familial qu'à réfléchir, à partir de cette histoire terrifiante et formidablement scénarisée, aux premiers mots qui, avant toute articulation conceptuelle, me sont venus à l'esprit à sa réécoute: regard, haine, et emprise.

\section{Regard}

Ce film en est d'abord un sur le regard. Regard d'un enfant qui, de sa prime jeunesse aux débuts de l'âge adulte, ne cesse de chercher, avec une intensité effrayante, celui de sa mère, et qui y reste fixé. Regard à la fois stupéfait, angoissé et opaque d'une mère qui n'arrive pas à voir suffisamment d'elle-même dans le regard de son bébé, de son enfant, de son adolescent. Regard voilé d'un père qui ne voit dans celui de son fils qu'une projection idéalisée de lui-même, c'est-à-dire le papa parfait, et qui ne voit dans celui de sa conjointe que ce qu'il se refuse à voir en lui-même. Regard totalement idéalisé d'une petite soeur qui ne veut voir dans celui que lui porte son frère qu'une réponse à son immense désir d'amour. Regard horrifié du monde devant un adolescent monstrueusement triomphant après son geste meurtrier. Et enfin, regard de mépris haineux porté par la communauté sur celle qui a enfanté un pareil monstre.

Freud, nous rappelle Jean-Claude Rolland (2006), accordait une importance considérable au regard, à la vue et à leurs attributs dans l'activité psychique et notamment dans l'instauration de la subjectivité. Mais c'est surtout au texte de Winnicott, Le rôle de miroir de la mère et de la famille dans le développement de l'enfant (1971), sans doute l'un de ses plus connus, que j'ai été ramené en pensant à la fonction du regard. Winnicott écrit:

Que voit le bébé quand il tourne son regard vers le visage de la mère? Généralement, ce qu'il voit, c'est lui-même. En d'autres termes, la mère regarde le bébé et ce que son visage exprime est en relation directe avec ce qu'elle voit (p. 155).

Jung et Roussillon (2013) précisent que si le bébé peut se voir dans le visage maternel, c'est à condition que la mère reflète son bébé et qu'elle l'investisse comme un double, "un double de soi, un autre semblable, à la fois même et différent de soi, un double transitionnel» (p. 1043). C'est sur le modèle winnicottien du trouvé-créé que l'investissement de ce double 
permettra la construction de la réflexivité interne, c'est-à-dire «comment un sujet se voit et se sent, comment il se pense et se représente son propre fonctionnement psychique, comment il réfléchit et se refléchit à lui-même ses propre expériences, et comment il se construit psychiquement à partir de ces différentes opérations» (p. 1043). Mais revenons au texte de Winnicott:

J'évoquerai, dit-il, le cas du bébé dont la mère ne reflèterait que son propre état d'âme ou, pis encore, la rigidité de ses propres défenses. Dans un cas semblable, que voit le bébé? (p. 155).

On peut en effet se demander, très tôt dans le film, ce que voit Kevin dans le regard de sa mère, ou ce qu'il ne voit pas? Et surtout, qu'est-ce qu'il continue à y chercher, en s'y accrochant au point de ne jamais cesser de le scruter, «tout comme nous scrutons le ciel pour deviner le temps qu'il va faire» dit encore Winnicott (p. 156). Il ajoute que chez le bébé qui regarde mais ne se voit pas lui-même dans le regard de sa mère, la capacité créative, qui permet de se sentir réel, en trouvant un moyen d'exister soi-même pour se sentir relié aux objets en tant que soi-même et pour avoir «un soi où se réfugier pour se détendre» (p.161), cette capacité créative, donc, va s'atrophier, et l'enfant va chercher un autre moyen pour que l'environnement lui reflète quelque chose de lui-même.

Pour Jung (2012), il s'agit alors d'un échec de l'expérience du trouvél créé, quand mère et bébé ne parviennent pas, dans la rencontre, à créer une illusion subjectivante, matrice de la fonction réflexive, ce miroir psychique interne par lequel le sujet pourra en venir à se représenter lui-même en l'absence de l'objet (ce qui, nous le verrons, ne deviendra jamais possible pour Kevin). Se produira plutôt une illusion négative, par laquelle l'enfant vivra son expérience comme produisant du négatif et qui ne permettra pas l'établissement d'une identité subjectivante (Roussillon, 1999).

L'atrophie de la capacité créative de Kevin et de son identité comme sujet nous est montrée de différentes façons dans le film, mais d'une manière tout à fait saisissante par les images de sa chambre d'adolescent, parfaitement rangée et vide, en total contraste avec celles de la plupart des adolescents, qui y déploient tous les signes de leurs investissements du monde et d'euxmêmes. Ici, on perçoit plutôt la monotonie et la répétition qui caractérisent la fixité mortifère de la perversion (Seulin, 2014).

Ce défaut de capacité créative laissera donc tout le champ libre au développement de la capacité destructrice. Ainsi, un des moyens auquel aura 
recours Kevin pour, comme le dit Winnicott « que l'environnement lui réfléchisse quelque chose de lui-même» (p. 155), consistera en l'attaque systématique de tout ce qui a le pouvoir «d'animer» le visage de la mère, d'éclairer son regard. Ainsi par exemple, sa joie débordante à voir son fils, enfin, lui retourner le ballon; ou encore son plaisir à décorer, dans une maison qu'elle n'a pas choisie et qu'elle déteste, une pièce personnelle, lieu où elle peut se retrouver, entourée des cartes géographiques témoignant d'une vie antérieure plus épanouissante ou qui du moins offrait des possibilités de fuite, elle qui se sent totalement prise au piège dans sa vie actuelle.

Et puis, le moyen ultime: l'attaque meurtrière contre des élèves de son école sur qui les autres élèves portent des regards admiratifs ou envieux, et leurs parents un regard bienveillant, fier et plein d'espoir. Geste irréparable, grandiose et hautement médiatisé, encore une fois motivé par la recherche du regard de la mère et, par extension, celui de la société toute entière.

Le film nous donne à voir que dès le départ quelque chose ne fonctionne pas dans la relation entre Kevin et sa mère. Il y a comme un ratage initial, un échec fondateur. Quelques lunettes conceptuelles nous sont disponibles pour en faire l'analyse. Ainsi, avec Wilfred Bion (1961, 1962, 1963, 1967): échec de la capacité de rêverie maternelle à contenir les éprouvés de son bébé et à transformer les éléments beta en éléments alpha; avec Winnicott (1956) : échec de la préoccupation maternelle primaire; avec Daniel Stern (1985): échec de l'accordage émotionnel entre mère et bébé; avec Donald Meltzer (2013): échec de l'expérience esthétique chez le nourrisson; avec René Roussillon $(2008,2010)$ : échec du plaisir partagé et de l'homosexualité primaire en double avec dépendance au plaisir mais sans expérience de satisfaction; avec, chez nous, Wilfrid Reid (2014) : échec de l'objet à favoriser le caractère vital plutôt que destructeur du mouvement pulsionnel original du sujet, et échec de la recherche par l'enfant, dans le regard de sa mère, d'un frein à son omnipotence. On pourrait bien sûr ajouter à la liste Jean Laplanche et les messages énigmatiques de la mère, ainsi que plusieurs autres.

En outre, on doit tout autant parler ici de l'échec total de la fonction paternelle auprès de la dyade mère-bébé, de par l'absence troublante de soutien offert à cette dernière par le père, qui ne la croit pas et, plus encore, désavoue complètement ce qu'elle vit et éprouve auprès de son fils. De par également, comme on l'a dit plus haut, son incapacité à voir autre chose dans son fils qu'une projection idéalisée de lui-même. Freud, dans son Léonard (1910), dit comment à l'arrivée d'un enfant, et surtout un fils, le père sent que celui-ci est devenu son rival, et qu'une profonde hostilité inconsciente 
prend alors naissance contre celui qui est vu comme le préféré. N'est-ce pas cette hostilité qui, chez le père de Kevin, est retournée en son contraire (le père n'est-il pas en effet d'une exaspérante gentillesse avec lui?), l'empêche de voir celle qu'il y a chez son fils et qui, massivement projetée, est toute entière attribuée à sa conjointe?

À propos justement de cette gentillesse du père, une phrase dite par Kevin et qu'on retrouve non dans le film mais dans le roman dont il est inspiré (Shriver, 2003) est très parlante: «Ça veut dire quoi un père qui t’aimet'aime-t'aime et qui n'a pas la moindre idée de qui tu es? Il aime quoi, en fait? Un môme de la série Happy Days. Pas moi» (p. 537). Un objet parfait, a dit quelque part Winnicott, ne vaut pas mieux qu'une hallucination. J'ajouterais pour ma part: un père parfait, c'est parfaitement inutile.

Mais que va-t-il donc résulter de cette relation de départ ratée entre Kevin et sa mère, de cet échec fondateur? Qu'est-ce qui va caractériser cet attachement démesuré à elle (a-t-il en effet un autre objet que celle-ci), ce lien passionnel (a-t-il un autre centre d'intérêt que les réactions émotionnelles qu'il peut provoquer chez elle)? Il semble que ce soit, d'après ce que le film nous donne à voir, la haine et l'emprise.

\section{Haine}

Dans un texte intitulé «S'exalter dans la haine», Paul Denis écrit: «La haine n'est pas plus le contraire de l'amour que le vinaigre n'est le contraire du vin. On pourrait tout à fait considérer que la haine est une forme d'amour. Elle est en tout cas une passion, et très souvent une forme de lien à l'objet.» (2013, p. 23). Il dit plus loin: «Si l'objet est connu dans la haine - Denis fait ici référence à la citation attribuée à Freud — la haine est une façon de le maintenir» (p. 24).

Mais ce lien à l'objet, basé sur la haine, a la caractéristique d'être très focalisé, puisque si l'amour peut avoir plusieurs objets, la passion amoureuse ou la haine ne peuvent n'en avoir qu'un seul. Denis ajoute: «Ce type d'investissement réduit toutes les dimensions de l'objet à celle d'une cible, il est traité comme un objet partiel dont il faut s'assurer la possession» (p. 28).

Ainsi, on voit bien chez Kevin cet investissement massif et focalisé de la mère, objet partiel réduit à la dimension du regard, dont le but est de s'en assurer la possession, et ce au détriment de tout autre investissement. Kevin n'a en effet aucune relation significative en dehors de sa mère, aucune activité créatrice, aucune passion, à l'exception du tir à l'arc, dont on verra que celle-ci est encore totalement arrimée à l'objet maternel. 
À cet égard, il est intéressant de lire, dans le roman de Shriver, la façon dont Kevin choisit ses victimes à l'école, ce qui n'est pas très explicite dans le film: soigneusement sélectionnés, il s'agit de confrères et consoeurs de classe, ainsi que d'une enseignante, qui tous sont reconnus dans le milieu pour leur passion, artistique, sportive ou autre. C'est à cette vie créative, dont il est totalement dépourvu, que Kevin s'attaque de façon envieuse, la qualifiant de «stupide».

Si l'amour amène une extension du moi, la haine entraîne plutôt une restriction, une concentration et une focalisation de celui-ci, un appauvrissement donc.

La haine, par ailleurs, est un moyen de lutte contre des angoisses de morcellement, d'effondrement, de dépersonnalisation. Elle devient nécessaire lorsque ces angoisses menacent l'unité du moi et annoncent une désorganisation psychique, une perte identitaire. L'exaltation dans la haine, dit Paul Denis, permet un sentiment de reconquête d'une unité et un pouvoir sur l'objet et sur soi-même.

Dans ce lien haineux qui unit Kevin et sa mère, aucune possibilité de satisfaction, ni pour l'un ni pour l'autre. Dans le roman, la mère dit: «Il me refusait manifestement et délibérément toute forme de satisfaction. Il avait décidé que je devais me sentir inapte et inutile» (p. 299). La satisfaction laisse plutôt place à l'emprise.

\section{Emprise}

La pulsion d'emprise, qui vise l'appropriation de l'objet, apparait pour la première fois chez Freud dans les Trois essais sur la théorie sexuelle (1905). Il s'agit pour lui d'une pulsion non sexuelle, donc d'autoconservation, qui peut s'unir aux pulsions sexuelles, et être au service de la satisfaction. D'après la définition qu'en donnent Laplanche et Pontalis (1967) dans leur Vocabulaire, le but de cette pulsion est de dominer l'objet par la force, et ce en ayant recours à l'appareil d'emprise, c'est-à-dire le toucher, l'appareil musculaire, les organes des sens en général (et chez Kevin la vision en particulier). Après Freud, quelques auteurs ont développé cette notion de pulsion d'emprise: Roger Dorey (1981) a ainsi parlé de la « relation d'emprise». Jean Bergeret (1984) a élaboré la notion de «violence fondamentale» à partir de la pulsion d'emprise.

Paul Denis (1992), pour sa part, a proposé l'idée que la pulsion ellemême, dans son organisation constitutive, contient une dimension d'emprise et une dimension de satisfaction, ce qu'il appelle les deux «formants» 
de la pulsion. Le poids économique de ces deux formants peut varier d'un sujet à l'autre; ils peuvent même, comme dans le cas de Kevin, se dissocier. La satisfaction laisse alors place à la recherche constante d'un sentiment de possession et de triomphe sur l'objet, qui a notamment pour conséquence une incapacité de s'identifier à l'autre, et un profond isolement.

L'emprise qui se joue entre Kevin et sa mère nous est montrée dès le début du film: la mère met son visage dans l'eau, et celui de son fils se superpose au sien. Cette emprise est également représentée par la façon dont les scènes entre eux sont tournées: en plan très serré, contribuant à nous faire éprouver un véritable étouffement.

Par ailleurs, c'est lorsque Kevin comprend que sa mère va échapper à son emprise qu'il décide de commettre son geste meurtrier, mouvement d'emprise ultime. Il entend en effet les parents parler de séparation, et saisit que si cela se produit, sa mère vivra avec sa soeur et lui, avec son père. Se séparer de la mère, donc, et la laisser vivre en dehors de lui. Issue intolérable, qu'il va empêcher par son crime, en liant ainsi pour toujours sa mère à lui, triomphe de l'emprise et de l'exclusion de tout autre, de tout tiers. Après son geste sa mère, ayant perdu fille, mari, entreprise et vie sociale, se retrouvera totalement seule et entièrement consacrée à Kevin, à ses visites à la prison, à l'attente de son retour chez elle, à la recherche d'une compréhension de son geste.

Mais même à travers cette relation d'emprise sur l'objet maternel, Kevin n'abandonnera jamais sa quête, celle d'un regard sur lui, dans lequel il pourrait se voir et se sentir exister. Que cherche-t-il de lui dans le regard de sa mère? Serait-ce le reflet de sa propre haine?

J'apporterai ici une courte vignette clinique:

Au cours de la dernière année, une patiente arrive à sa séance dans un état proche de la détresse. Elle se dit totalement dépassée par les comportements opposants de son fils âgé de deux ans, qui à moi pourtant m'apparaissent plutôt de bon augure pour son développement psychique. La patiente se montre d'abord écrasée par la culpabilité: si son fils est à ce point difficile, c'est à cause d'elle, de ses tourments d'ordre amoureux qui la rendent moins disponible psychiquement, ce que son fils ne peut que ressentir et à quoi il réagit nécessairement. Puis, derrière la culpabilité apparait l'angoisse: elle est convaincue que son fils développe un "trouble oppositionnel avec provocation", qu'il va donc 
être problématique à l'école, va prendre les études en grippe, être intolérant à toute autorité et devenir... un petit Kevin, peut-être? Un peu plus et elle se voit déjà lui rendre visite à son centre jeunesse.

La patiente me demande, désemparée, ce qu'elle doit faire, quel spécialiste consulter, pour finir par me dire que ce qu'elle craint le plus c'est de cesser d'aimer son fils. Je me permets alors de lui dire qu'effectivement, son petit, actuellement, est haïssable, et que je comprends qu'elle puisse le haïr... tout en n'arrêtant pas pour autant de l'aimer. Cette intervention très banale ${ }^{3}$ a pourtant un effet interprétatif chez elle. Dans les jours qui suivent la séance, elle décide de parler à son fils de ses comportements détestables, de ce qu'elle en comprend et ressent, tout en lui accordant un peu plus de temps pour jouer avec elle. Les choses rentrent rapidement dans l'ordre: apaisée, adoptant une autre attitude face à l'opposition de son garçon, celui-ci à son tour s'apaise. Étant bloquée, en raison de sa propre histoire, dans la reconnaissance du mouvement haineux en elle, par crainte qu'il n'emporte avec lui tout lien d'attachement et d'amour, elle n'arrivait pas à être pour son fils un miroir pour sa colère et sa demande avide de présence maternelle. Peut-être avions-nous ici, pour citer à nouveau Winnicott, "le cas du bébé dont la mère ne réfléterait que son propre état d'âme...».

Le roman duquel est tiré le film, davantage que le film lui-même, nous donne quelques clés pour comprendre ce qui, chez la mère de Kevin, voile son regard et l'empêche d'être un miroir adéquat. D'une part, la profonde monotonie de son monde intérieur, l'amenant à voyager de façon compulsive autour du monde, à la recherche d'une expérience de transformation. D'autre part, sa terreur à l'idée d'avoir un enfant et de se «retrouver désespérément coincée dans l'histoire de quelqu'un d'autre» (p. 57). D'être sous l'emprise d'un autre, donc. Enfin, la présence en elle d'une injonction la forçant à taire la haine, qui l'empêchera plus tard de contenir celle de son fils. Il est intéressant ici de souligner que la mère va insister pour donner son nom de famille à Kevin (alors qu'elle donnera à sa fille le nom du père), et ce afin de contrer l'impression qu'il lui est étranger. Ce nom est celui de son propre père, mort à la guerre pendant que sa mère était enceinte d'elle. Et c'est un nom arménien, évocateur pour elle de la haine ayant conduit au génocide de son peuple.

Mais à deux moments dans le film, l'espoir d'une relation différente entre Kevin et sa mère apparaîtra.

D'abord, celui où, malade, il laissera sa mère être une mère, le prendre dans ses bras et lui lire des histoires. On sait par ailleurs quelle trace ce 
moment de rapprochement laissera en lui lorsque s'identifiant au Robin des Bois de l'histoire lue par maman, il en deviendra une version perverse, grandiose et destructrice.

L'autre moment, lorsque celle-ci n'en peut plus de le voir résister à devenir propre, et lui exprime sa rage par un geste violent. Je cite ici deux phrases du roman: «Pour une fois, je me suis sentie pleine de grâce, parce qu'enfin existait une convergence non médiatisée entre ce que je pensais et ce que je faisais» (p. 303). Et: «Le véritable amour a plus en commun avec la haine et la colère qu'avec la bienveillance ou la politesse. Pendant deux secondes, j'avais senti que j'étais à la fois moi-même et la vraie maman de Kevin Khatchadourian. J'étais proche de lui. J'étais moi-même dans sa vérité non-expurgée - et nous communiquions enfin» (p. 303).

Après ce geste de colère, inscrivant la castration anale ${ }^{4}$, Kevin devient propre. Et quelques années plus tard, en prison, montrant encore une fois sa cicatrice à sa mère, il évoquera cet incident en disant: "C'est la seule chose sincère que tu as faite». Mais entre-temps, cette blessure et surtout le secret qui va l'entourer, scellant un pacte entre Kevin et sa mère, se mettra elle aussi au service de l'emprise.

Puis, à la fin du film, une porte ouverte sur un possible changement dans le rapport qu'entretient Kevin à lui-même. En réponse à sa mère qui lui demande pourquoi il a commis le geste qui l'a conduit en prison, Kevin répond: "Avant je savais... maintenant je n'en suis plus sûr... ». Son regard alors n'en est plus un de défi, de vengeance et de haine, et est troublé par le doute, davantage tourné vers lui-même que vers l'extérieur, vers la mère. Espoir d'un début d'instauration de réflexivité? D'un renoncement possible au triomphe narcissique palliant la faillite de la constitution du narcissisme (Seulin, 2014)?

Je terminerai en citant à nouveau un passage du roman, celui où la mère repense au moment où elle voit son fils la regardant à travers la glace de la voiture de police, tout juste après son arrestation: «Pourtant, quand je revois aujourd'hui son visage derrière cette vitre arrière, je me souviens d'autre chose. Une recherche. Il cherchait quelque chose sur mon visage. Il l'a scruté longuement et attentivement, avant de s'adosser de nouveau sur la banquette. Quel qu'ait été l'objet de sa quête, il n'avait pas trouvé, ce qui a aussi paru, d'une certaine façon, le satisfaire. Il n'a pas souri. Mais il aurait fort bien pu» (p. 580).

Winnicott parlait du travail du psychothérapeute comme d'un dérivé complexe du visage qui réfléchit ce qui est là pour être vu. Mais, nous 
rappelle Jean Claude Rolland, «L'image que l'analyste renvoie à l'analysant est une image vivante s'animant de ce que la rencontre produit de changement chez les deux protagonistes de la situation. Elle n'est pas pur reflet» (2006, p. 149). C'est à cette condition que le patient, et peut être aussi le thérapeute, pourra se sentir réel et «trouver un moyen d'exister soi-même, pour se relier aux objets en tant que soi-même et pour avoir un soi où se réfugier afin de se détendre» (Winnicott, 1975, p. 161).

\section{Réal Laperrière laperrierereal@videotron.ca}

\section{Notes}

1. Merci à Marcel Hudon, psychiatre et psychanalyste, de m’avoir signalé cette citation.

2. Le Ciné-Psy est un événement parrainé par l'Association des psychothérapeutes psychanalytiques du Québec (APPQ), organisé par André Jacques, psychologue.

3. Mais dans le dispositif analytique aucune intervention ne peut être banale, puisque toujours liée au transfert.

4. Merci à Stephany Squires, doctorante en psychologie, pour m'avoir mis sur la piste de ce concept de Françoise Dolto.

\section{Références}

Bergeret, J. (1984). La violence fondamentale. Paris: Dunod.

Bion, W. R. (1961). Recherches sur les petits groupes. Paris: Presses universitaires de France, 1965.

Bion, W. R. (1962). Aux sources de l'expérience. Paris: Presses universitaires de France, 1979.

Bion, W. R. (1963). Éléments de la psychanalyse. Paris: Presses universitaires de France, 1979.

Bion, W. R. (1967/1974). Différenciation de la part psychotique et de la part non psychotique de la personnalité. Nouvelle Revue de psychanalyse, $\mathrm{n}^{\circ}$ 10, 61-78.

Denis, P. (1992). Emprise et satisfaction. Paris: Presses universitaires de France.

Denis, P. (2013). De l'exaltation. Paris: Presses universitaires de France.

Dorey, R. (1981). La relation d'emprise. Nouvelle Revue de psychanalyse, nº 24, 117-140.

Ferenczi, S. (1929). L'enfant mal accueilli et sa pulsion de mort. Dans Euvres complètes IV. Paris: Payot, 1982.

Freud, S. (1905). Trois essais sur la théorie sexuelle. Paris: Gallimard, 1962.

Freud, S. (1910). Un souvenir d'enfance de Léonard de Vinci. Paris: Gallimard, 1987.

Jung, J. (2012). Le double transitionnel. Trajectoire identitaire et organisation réflexive. Thèse de doctorat de Psychologie, Université Lumière Lyon 2.

Jung, J., Roussillon, R. (2013). L’identité et le double transitionnel, Revue française de psychanalyse, 77 (4), 1042-1054.

Laplanche, J.; Pontalis, J.-B., (1967). Vocabulaire de la psychanalyse. Paris: Presses universitaires de France

Matot, J.-P., Roussillon, R. (2010). La psychanalyse: une remise en jeu. Paris: Presses universitaires de France.

Meltzer, D. (2013). Sur l'objet et le conflit esthétique. Journal de la psychanalyse de l'enfant, 3 (1), 29-39. 
Reid, W. (2014). L'utilisation de l'objet: Winnicott et la pensée ternaire. Texte présenté à la Société psychanalytique de Montréal.

Rolland, J.-C. (2006). Avant d'être celui qui parle. Paris: Gallimard.

Roussillon, R. (1999). Agonie, clivage et symbolisation. Paris: Presses universitaires de France.

Roussillon, R. (2008) Le jeu et l'entre-je(u). Paris: Presses universitaires de France

Seulin, C. (2014/2015). Émergence et transformations de la sexualité infantile dans la cure. Bulletin de la société psychanalytique de Paris, $\mathrm{n}^{\circ}$ 1, 33-106.

Shriver, L. (2003). Il faut qu'on parle de Kevin. Paris: Belfond, 2006.

Stern, D. (1985). Le monde interpersonnel du nourrisson Paris: Presses universitaires de France, 1989.

Winnicott, D.W. (1956). La préoccupation maternelle primaire. Dans De la pédiatrie à la psychanalyse (p. 285-291). Paris: Payot, 1969.

Winnicott, D. W. (1971). Le rôle de miroir de la mère et de la famille dans le développement de l'enfant. Dans Jeu et réalité. L'espace potentiel (p. 153-162). Paris: Gallimard, 1975. 